\title{
Mediators of the association between pre-eclampsia and cerebral palsy: population based cohort study
}

\author{
@) (1) $\Theta$ OPEN ACCESS
}

\author{
Kristin Melheim Strand medical student ${ }^{1}$, Runa Heimstad senior consultant in obstetrics and \\ gynaecology ${ }^{2}$, Ann-Charlotte Iversen senior researcher ${ }^{1}$, Rigmor Austgulen professor of paediatrics ${ }^{1}$, \\ Stian Lydersen professor of medical statistics ${ }^{3}$, Guro L Andersen senior consultant in paediatrics ${ }^{4}$, \\ Lorentz M Irgens professor of preventive medicine ${ }^{5}$, Torstein Vik professor of paediatrics ${ }^{6}$
}

\begin{abstract}
'Department of Cancer Research and Molecular Medicine, Norwegian University of Science and Technology (NTNU), PO Box 8905, MTFS, N-7491 Trondheim, Norway; ${ }^{2}$ Department of Obstetrics and Gynecology, St Olav's University Hospital, Trondheim, Norway; ${ }^{3}$ The Regional Centre for Child and Adolescent Mental Health, NTNU, Trondheim, Norway; ${ }^{4}$ Norwegian Cerebral Palsy Registry, Habilitation Center, Vestfold Hospital, Tønsberg, Norway; ${ }^{5}$ Medical Birth Registry of Norway, Locus of Registry Based Epidemiology, Department of Public Health and Primary Health Care, University of Bergen and the Norwegian Institute of Public Health, Norway; ${ }^{6}$ Department of Laboratory Medicine, Children's and Women's Health, NTNU, Trondheim, Norway
\end{abstract}

\begin{abstract}
Objective To test the hypothesis that pre-eclampsia is a risk factor for cerebral palsy mediated through preterm birth and being born small for gestational age.

Design Population based cohort study.

Setting Clinical data from the Norwegian Cerebral Palsy Registry were linked with perinatal data prospectively recorded by the Medical Birth Registry of Norway.

Participants All singleton babies who survived the neonatal period during 1996-2006 (849 children with cerebral palsy and 616658 control children).

Main outcome measures Cerebral palsy and cerebral palsy subtypes.

Results Children exposed to pre-eclampsia had an excess risk of cerebral palsy (unadjusted odds ratio $2.5,95 \%$ confidence interval 2.0 to 3.2) compared with unexposed children. Among children born at term ( $\geq 37$ weeks), exposure to pre-eclampsia was not associated with an excess risk of cerebral palsy in babies not born small for gestational age (1.2, 0.7 to 2.0$)$, whereas children exposed to pre-eclampsia and born small for gestational age had a significantly increased risk of cerebral palsy (3.2, 1.5 to 6.7). Non-small for gestational age babies born very preterm (<32 weeks) and exposed to pre-eclampsia had a reduced risk of cerebral palsy compared with unexposed children born at the same gestational age $(0.5,0.3$ to 0.8$)$, although the risk was not statistically significantly reduced among children exposed to pre-eclampsia and born small for gestational age $(0.7,0.4$ to 1.3$)$. Exposure to pre-eclampsia was not associated with a specific cerebral palsy subtype.
\end{abstract}

Conclusions Exposure to pre-eclampsia was associated with an increased risk of cerebral palsy, and this association was mediated through the children being born preterm or small for gestational age, or both. Among children born at term, pre-eclampsia was a risk factor for cerebral palsy only when the children were small for gestational age.

\section{Introduction}

Cerebral palsy includes a group of permanent disorders of movement or posture caused by an early brain injury. ${ }^{1}$ Although several factors, including preterm birth and low birth weight for gestation, ${ }^{2}$ are associated with excess risk, the causes of cerebral palsy remain largely unknown. Pre-eclampsia affects $3-5 \%$ of pregnant women and is characterised by maternal hypertension and proteinuria occurring after 20 weeks of gestation. ${ }^{4}$ Serious manifestations may induce iatrogenic preterm delivery, and pre-eclampsia contributes substantially to prematurity, perinatal morbidity, and mortality. ${ }^{4}$ Early onset pre-eclampsia (before 34 weeks of gestation) is commonly associated with severe placental dysfunction, which can compromise fetal blood supply and cause fetal growth restriction, ${ }^{5}$ chronic hypoxaemia, and possibly brain damage. ${ }^{6}$ Hence it is plausible that pre-eclampsia could be a risk factor for cerebral palsy.

Some studies have found an excess risk of cerebral palsy in children born at term from pre-eclamptic pregnancies, ${ }^{6-10}$ whereas others have reported no association. ${ }^{11-13}$ Recently, researchers found an excess risk of cerebral palsy in children from 
pre-eclamptic pregnancies diagnosed before 37 weeks of gestation, and they concluded that while some of the association probably was attributable to preterm birth, a direct effect was likely. ${ }^{7}$ The authors did not, however, attempt to explore whether the effect at least partly could be ascribed to the fetal growth restriction often accompanying pre-eclampsia. Furthermore, no study to date has assessed whether pre-eclampsia is associated with specific subtypes of cerebral palsy. Pre-eclampsia is considered a vascular endothelial disorder and has been identified as an independent risk factor for perinatal arterial ischaemic stroke. ${ }^{14}$ Cerebral palsy, and especially unilateral cerebral palsy in children born at term, is considered a common outcome of perinatal stroke, ${ }^{15}$ and a causal relation between pre-eclampsia and unilateral cerebral palsy might therefore be plausible. Hence, a specific cerebral palsy subtype would be expected to be more common in children from pre-eclamptic pregnancies if pre-eclampsia has a direct effect on the risk of cerebral palsy.

In the present study, we linked data from the Norwegian Cerebral Palsy Registry with data from the Medical Birth Registry of Norway to test the hypothesis that pre-eclampsia is a risk factor for cerebral palsy mediated through preterm birth and being born small for gestational age. Finally, we hypothesised that a direct effect of pre-eclampsia on the risk of cerebral palsy would result in a specific cerebral palsy subtype, and in particular the unilateral spastic cerebral palsy subtype.

\section{Methods}

All singleton babies surviving the early neonatal period in Norway between 1 January 1996 and 31 December 2006 were eligible for this study. From the Medical Birth Registry of Norway we extracted data on pre-eclampsia in pregnancy, maternal health and delivery, and the early neonatal period. Using the 11 digit person identification number unique for each Norwegian citizen, we linked these data with clinical data recorded in the cerebral palsy registry, collected through all paediatric habilitation centres in Norway between 1 January 2003 and 27 March 2012. The recording of data in the cerebral palsy registry of Norway and linkage with the medical birth registry requires informed consent from the parents. In addition to this detailed consent based information, the habilitation centres report the total number of children with cerebral palsy for whom they care (summative information).

\section{Study population}

A total of 646731 children were born between 1996 and 2006. We excluded 6905 neonates registered as stillbirths or dead in the first week of life, and 78 neonates born before 22 weeks of gestation (see supplementary figure $1 \mathrm{~b}$ ).

According to the summative information from the paediatric habilitation centres, 1494 children born between 1996 and 2006 had a diagnosis of cerebral palsy. By 27 March 2012, detailed data for 381 of these children $(25.5 \%)$ had not been recorded in the cerebral palsy registry (see supplementary figure 1a). Since no information was available on these children, including birth dates, we were not able to exclude these children from the reference population. Among the remaining 1113 children with detailed clinical information, we excluded 80 because of missing data in the birth registry ( 70 children were born abroad and 10 were not registered in the birth registry). Forty nine children with cerebral palsy of postneonatal origin were excluded. We finally excluded 22112 children born of multiple pregnancies, 135 of whom had cerebral palsy. Thus the final study population included 849 children with cerebral palsy (1.4 per $1000,58.7 \%$ boys and $41.3 \%$ girls) and 616658 children without cerebral palsy.

\section{Study variables}

Cerebral palsy was diagnosed and classified according to the Surveillance of Cerebral Palsy in Europe guidelines. ${ }^{16}$ The cerebral palsy registry of Norway is an informed consent based registry containing detailed data on each registered child, provided by all paediatric habilitation centres in Norway. A senior paediatrician records the clinical data on standardised forms. ${ }^{17}$ The diagnosis of cerebral palsy was confirmed in all the children when they were at least four years old, according to the recommendations of the Surveillance of Cerebral Palsy in Europe network. ${ }^{16}$ The distribution of cerebral palsy subtypes and gross or fine motor functions did not differ when comparing the characteristics of the children with cerebral palsy between habilitation centres with nearly complete recording of cases ( $>90 \%$ of cases) and habilitation centres with low participation ( $<90 \%$ of cases).

We retrieved data on pre-eclampsia in pregnancy from the Medical Birth Registry of Norway. Since 1967 the registry has collected data prospectively on all births in Norway through compulsory notification. ${ }^{18}$ Until 2006, obstetric guidelines in Norway defined pre-eclampsia as a rise in blood pressure after 20 weeks of gestation to $\geq 140 / 90 \mathrm{~mm} \mathrm{Hg}$, a rise in systolic blood pressure of $\geq 30 \mathrm{~mm} \mathrm{Hg}$ from baseline (that is, blood pressure measured before the 20th week of gestation), or a rise in diastolic blood pressure of $\geq 15 \mathrm{~mm} \mathrm{Hg}$ from baseline, combined with proteinuria ( $\geq 0.3 \mathrm{~g} / 24 \mathrm{~h}$ urine or $\geq+$ dipstick). ${ }^{19}$ Since 1 January 1999, pregnancies registered in the birth registry by checkbox with pre-eclampsia, early onset pre-eclampsia (onset before 34 weeks), eclampsia, or HELLP syndrome (haemolysis, elevated liver enzymes, and low platelet count syndrome) were defined as pre-eclamptic. Before 1999 pre-eclampsia was reported as "complications" under the item "Mother's health during pregnancy" and specified as pre-eclampsia in free text.

From the birth registry we obtained data on the mothers (age at delivery), pregnancies (parity, gestational age at birth, multiple pregnancies), and neonates (sex, birth weight, Apgar score). We dichotomised women's parity as nulliparous (no previous births) or parous ( $\geq 1$ previous births). In the registry, gestational age at birth is calculated from routine ultrasonographic measurements of biparietal diameter at 17-18 weeks of gestation according to Norwegian growth curves. If ultrasonographic measurements are missing, gestational age is calculated from the first day of the last menstrual period. We divided duration of pregnancy into three categories: term birth $(\geq 37+0$ weeks of gestation), moderate preterm birth ( $32+0$ to $36+6$ weeks), and very preterm birth ( $\leq 31+6$ weeks). We used small for gestational age as a proxy for fetal growth restriction and defined small for gestational age as a birth weight below the 10th centile, calculated according to Norwegian sex and gestational age specific standard curves. ${ }^{20}$ Since $3.1 \%$ of the children had missing data on gestational age, the number of participants in the analyses including children born small for gestational age as well as in the analyses stratified according to duration of pregnancy are lower than in the total study population.

Other covariates retrieved from the birth registry included assisted fertilisation (in vitro fertilisation or intracytoplasmatic sperm injection) and initiation of delivery (spontaneous, medically induced, or by caesarean section). Since 1999 the birth registry has also recorded information on smoking at the beginning or towards the end of pregnancy. The recording of 
this information required informed consent by the mothers; 350 $865(56.8 \%)$ women had information on smoking. The proportion of mothers with information on smoking did not differ between children with cerebral palsy $(n=492,58.0 \%)$ and without cerebral palsy ( $\mathrm{n}=350373,56.8 \%)$, whereas this proportion was slightly higher in pre-eclamptic pregnancies $(n=13794,60.1 \%)$ than in non-pre-eclamptic pregnancies $(\mathrm{n}=227071,56.8 \%)$.

\section{Statistical analyses}

We used SPSS (version 20) for statistical analyses. Differences in proportions were analysed using $\chi^{2}$ statistics. We used logistic regression to analyse the effect of pre-eclampsia on cerebral palsy. In adjusted analyses we took into account the hierarchical relation between the suggested risk factors (see supplementary figure 2).$^{21}$ Firstly, we adjusted for factors considered to be possible mediators of the potential effect of pre-eclampsia on cerebral palsy ${ }^{22}$; namely, small for gestational age (model 2) and preterm birth (model 3). Furthermore, we included an interaction term of each potential mediator and pre-eclampsia. ${ }^{23}$ If the interaction term was statistically significant, we included it in the model (model 4). Finally, we adjusted for potential confounders. We considered a variable to be a potential confounder if it could theoretically be associated with both exposure (pre-eclampsia) and outcome (cerebral palsy) and could not be affected by exposure or outcome. We first explored separately the effects of these potential confounders, including maternal age, parity, smoking in pregnancy, assisted fertilisation, and sex of the child, in that order. We a priori decided to include only those variables in the final model that significantly affected the association between pre-eclampsia and cerebral palsy-that is, changed the unadjusted odds ratio by more than $10 \%$.

To further explore the role of small for gestational age as a potential mediator of the effect of pre-eclampsia on cerebral palsy, we compared the individual and combined effects of pre-eclampsia and small for gestational age on the risk of cerebral palsy.

Where relevant we report $95 \%$ confidence intervals. We considered two sided $\mathrm{P}$ values lower than 0.05 to be significant.

\section{Results}

Table $1 \Downarrow$ presents the characteristics of the mothers and children within the study groups.

Children exposed to pre-eclampsia had an excess risk of cerebral palsy compared with non-exposed children (unadjusted odds ratio $2.52,95 \%$ confidence interval 1.98 to 3.19 , table $2 \Downarrow$ ). Adjusting for small for gestational age reduced the unadjusted odds ratio of cerebral palsy after exposure to pre-eclampsia to 2.14 (1.67 to 2.74, table 2, model 2). When we further adjusted for duration of pregnancy, children exposed to pre-eclampsia had an odds ratio for cerebral palsy of 0.73 ( 0.56 to 0.96 , model $3)$. However, although there was no significant interaction between pre-eclampsia and small for gestational age on risk of cerebral palsy $(\mathrm{P}=0.17)$, there was a statistically significant interaction between pre-eclampsia and duration of pregnancy on risk of cerebral palsy $(\mathrm{P}=0.002)$. This indicates that the effect of pre-eclampsia on cerebral palsy varies with duration of pregnancy. Consequently, table $3 \Downarrow$ shows the risk for cerebral palsy in children exposed to pre-eclampsia who were or were not small for gestational age stratified by duration of pregnancy. In term children, pre-eclampsia alone was not associated with an excess risk of cerebral palsy (table 3), whereas if the child was also born small for gestational age the odds ratio of cerebral palsy was 3.18 (1.50 to 6.71). A similar pattern was seen among children born moderately preterm (table 3), although the odds ratio for cerebral palsy in children born small for gestational age and exposed to pre-eclampsia was not statistically significantly increased (1.59, 0.80 to 3.16$)$. In children born very preterm, pre-eclampsia alone was associated with a reduced risk of cerebral palsy, whereas if the exposed child also was small for gestational age, the risk for cerebral palsy was not statistically different compared with unexposed children born at the same duration of pregnancy (table 3).

To study the overall risk for cerebral palsy in children exposed to pre-eclampsia allowing for the interaction with duration of pregnancy, we constructed a new variable with six categories, combining pre-eclampsia (yes or no) and duration of pregnancy (term, moderately preterm, very preterm). Using children unexposed to pre-eclampsia and born at term as the reference group we assessed the risk of cerebral palsy in each category. Table $4 \Downarrow$ shows that in a model adjusting for small for gestational age status children born at term and exposed to pre-eclampsia did not have an excess risk for cerebral palsy, whereas children born moderately preterm had a nearly fivefold excess risk, and children born very preterm and exposed to pre-eclampsia had a significantly increased risk (adjusted odds ratio $20.37,95 \%$ confidence interval 13.74 to 30.22 ). Table 4 also shows the adjusted odds ratio for cerebral palsy in children born small for gestational age in this model. These results did not change by adjusting for maternal age, parity, smoking in pregnancy, assisted fertilisation, or sex of the child.

\section{Pre-eclampsia and cerebral palsy subtypes}

Among children with cerebral palsy, the distribution of subtypes did not differ between exposed and unexposed children (data not shown). These results did not change in analyses restricted to preterm or term birth (data not shown). Furthermore, we analysed the risk of each individual cerebral palsy subtype after exposure to pre-eclampsia. Children exposed to pre-eclampsia had an increased risk of unilateral cerebral palsy (adjusted odds ratio $2.16,95 \%$ confidence interval 1.41 to 3.30 ), bilateral cerebral palsy $(3.02,2.13$ to 4.28$)$, and ataxic cerebral palsy $(2.88,1.03$ to 8.10$)$, but no increased risk of dyskinetic cerebral palsy $(1.32,0.41$ to 4.21$)$ compared with unexposed children (data not shown in tabular form).

\section{Discussion}

In this study we found that pre-eclampsia was associated with an increased risk of cerebral palsy and that the excess risk was mainly mediated through preterm birth, but also through being born small for gestational age. Exposed children born at term as non-small for gestational age did not have an excess risk of cerebral palsy and we did not find that a specific cerebral palsy subtype was more common in children exposed to pre-eclampsia than not exposed. Thus we were not able to find evidence for a direct effect of pre-eclampsia on the risk of cerebral palsy.

\section{Strengths and limitations of this study}

Strengths of our study include the population based design and the large sample size, the large number of obstetrical and perinatal information prospectively recorded, and the detailed information from the cerebral palsy registry. In general, the observed effects of pre-eclampsia on cerebral palsy in our study were strong. The possibility that chance may explain our results is small as indicated by the low $\mathrm{P}$ values and the large number of children included in most analyses. However, few children had a diagnosis of ataxic and dyskinetic cerebral palsy, and the results for these subgroups should be interpreted with caution. 
Furthermore, we did sensitivity analyses by excluding children born in 1996 to 1998 (different reporting of pre-eclampsia) and by excluding children with congenital malformations and including multiple gestations. These analyses did not change the results. Information bias is unlikely since data on pregnancy and the neonatal period were recorded prospectively in the medical birth registry and data on cerebral palsy were collected independently of the birth registry record when the children were at least 4 years old.

A limitation to our study is that 381 children with cerebral palsy were not included in the cerebral palsy register. This was mainly because of work overload in some of the paediatric habilitation centres and not because of low compliance; only 34 (3\%) parents did not wish to include their child in the cerebral palsy register. Since we found no differences in the distribution of cerebral palsy subtypes, gross or fine motor functions between habilitation centres with high or low participation, we consider these dropouts most likely to be at random. Furthermore, it is unlikely that exposure to pre-eclampsia would differ systematically between registered and non-registered children with cerebral palsy. ${ }^{17}$ Finally, the misclassification of these 381 children as non-cerebral palsy in our analyses is negligible in the total population of 616658 reference children. Of the 1113 children with cerebral palsy in the register, we excluded 264 owing to multiple gestations, postneonatal cause for cerebral palsy, and birth abroad. Comparing the children with cerebral palsy who were excluded and included in our final study group revealed no differences in cerebral palsy subtypes or severity. We therefore consider our study group to be representative of the total population of children born with cerebral palsy in Norway between 1996 and 2006.

We cannot exclude that pre-eclampsia was misdiagnosed in the medical birth registry. If present, such misclassification would reduce the odds ratios. However, a validation study for the years 1967-2005 found that $88.3 \%$ of pre-eclamptic pregnancies registered in the birth registry agreed with medical records. ${ }^{24}$ This study also validated the pre-eclampsia diagnosis in the birth registry to international diagnostic standards and found that in the years 1999 to $200582 \%$ of the cases fulfilled the international criteria. ${ }^{25} \mathrm{We}$ therefore consider it unlikely that such misclassification would explain our results. However, we cannot exclude that some milder cases of pre-eclampsia were not registered, potentially leading to an underestimation of the risk of cerebral palsy associated with exposure to pre-eclampsia in children born at term.

Another potential limitation is the use of small for gestational age as a proxy for fetal growth restriction. The 10th centile definition of small for gestational age, even when adjusted for parity and sex, is liable to include constitutionally small children in addition to those who are growth restricted, whereas some babies who have not reached their growth potential will have birth weights above this cut-off. Using a lower cut-off such as the first, third, or fifth centile might have reduced the misclassification of the constitutionally small children as growth restricted but at the cost of a significantly lower number of children who were born small for gestational age and a higher proportion of growth restricted children in the non-small for gestational age group. More sophisticated methods, such as the method proposed by Gardosi et al, could not be applied owing to essential information missing in the medical birth registry. ${ }^{26}$ None the less, misclassification of constitutionally small children as growth restricted, and vice versa, inherent in any birth weight based statistical cut-off is most likely to dilute the differences between small for gestational age and non-small for gestational age children. Thus the impact of being small for gestational age on the risk of cerebral palsy associated with pre-eclampsia is therefore most likely an underestimate of the effect of fetal growth restriction.

Multivariable analyses did not suggest confounding by maternal age, parity, smoking in pregnancy, assisted fertilisation, or sex of the child.

\section{Comparison with other studies}

Although pre-eclampsia is associated with an increased risk of fetal growth restriction and small for gestational age births, which in turn is associated with an excess risk of cerebral palsy, ${ }^{3-27}$ our study is apparently the first to report that the association between exposure to pre-eclampsia and cerebral palsy may be partly mediated through fetal growth restriction, assessed by the proxy small for gestational age. Moreover, we are not aware of previous studies exploring the association between exposure to pre-eclampsia and specific cerebral palsy subtypes.

The overall increased risk of cerebral palsy after exposure to pre-eclampsia, without consideration of duration of pregnancy, is in agreement with several, ${ }^{6-10}$ but not all, ${ }^{11-13}$ previous studies. In analyses restricted to term birth, the lack of an association between pre-eclampsia and cerebral palsy is in line with two large scale cohort studies. ${ }^{11}{ }^{12}$ However, two studies on children born at term found an increased risk of cerebral palsy associated with pre-eclampsia. ${ }^{6}{ }^{10}$ Both study designs were case-control that included fewer than 250 children with cerebral palsy. Thus, differences in results concerning children born at term could be explained by study design and study size.

Consistent with our findings, others have reported that very preterm children exposed to pre-eclampsia are at lower risk for cerebral palsy compared with unexposed children born at the same gestational age. ${ }^{2-28}$ Some authors have therefore suggested that pre-eclampsia may have a neuroprotective effect, ${ }^{29}$ whereas more recent studies have concluded that the apparent protective effect of pre-eclampsia on cerebral palsy in very preterm infants may be caused by pre-eclampsia having less detrimental effects on the fetal brain than some other causes of spontaneous preterm birth. ${ }^{9}$ In our study, among children with cerebral palsy born very preterm and unexposed to pre-eclampsia, $65.9 \%$ of mothers went into labour spontaneously. The corresponding proportion in children with cerebral palsy exposed to pre-eclampsia was $13.3 \%$. Several studies have discussed that cerebral palsy associated with very preterm birth in many cases may have the same cause as the preterm birth itself-for example, a low grade intrauterine infection rather than the consequence of being born very prematurely. ${ }^{30}$ In our study, very preterm small for gestational age children exposed to pre-eclampsia had a risk of cerebral palsy comparable to unexposed children born at the same duration of pregnancy. Although speculative, this may be compatible with the findings of McElrath et al who reported an increased risk of cranial ultrasound lesions in very preterm children delivered because of fetal growth restriction. ${ }^{31}$ It should be emphasised that although the risk of cerebral palsy in very preterm children exposed to pre-eclampsia is reduced compared with unexposed children born at the same gestational length, it is significantly increased compared with unexposed children born at term.

\section{Interpretation of the findings}

Firstly, our findings suggest that the association between pre-eclampsia and cerebral palsy is mainly mediated through preterm birth and being born small for gestational age. Both the lack of an association between exposure to pre-eclampsia and 
a specific cerebral palsy subtype and the lack of an excess risk of cerebral palsy in exposed non-small for gestational age children born at term, suggest that pre-eclampsia does not have a direct effect on the risk of cerebral palsy. Secondly, the lack of an excess risk of dyskinetic cerebral palsy with pre-eclampsia combined with the finding of no association between pre-eclampsia and low Apgar scores (data not shown), may suggest that a possible brain injury caused by pre-eclampsia would antedate delivery rather than arise through birth asphyxia. Several possible mechanisms exist by which pre-eclampsia could increase the risk of cerebral palsy. Most importantly pre-eclampsia is a frequent cause of preterm birth, known as the strongest predictor for cerebral palsy. Preterm delivery in pre-eclamptic pregnancies is mostly as a result of iatrogenic delivery of a baby to save the mother's life. Brain insults in very preterm born children exposed to pre-eclampsia may be caused by complications such as intracranial haemorrhages, ischaemic episodes, and infections during birth as well as in the neonatal period, as seen in spontaneously delivered unexposed children. However, intrauterine hypoxaemic episodes due to impaired placental function may also already have caused antenatal brain insults in these children, even in the absence of fetal growth restriction. The reduced risk of cerebral palsy among very preterm born exposed children compared with unexposed children may be explained by a higher prenatal and perinatal morbidity among spontaneous births, where perinatal infections are thought to play an important role.

In children born at term, the course of pre-eclampsia has often been milder, but when resulting in the birth of a small for gestational age infant a reasonable underlying mechanism may be poor placental function and chronic hypoxaemia leading to a brain insult. Thus we speculate that the excess risk of cerebral palsy associated with pre-eclampsia, in addition to the complications of prematurity, may arise through poor placental function with or without growth restriction. Supportive of our interpretation is the comprehension that the pre-eclamptic syndrome consists of several different entities, where the severe and early onset forms differ from late onset disease not just by affecting the mother severely but also by the serious impact on neonatal morbidity. It seems plausible that mild pre-eclampsia in late pregnancy would have little potential for fetal brain injury, whereas pre-eclampsia accompanied by small for gestational age reflects a longstanding ischaemic intrauterine environment, which well could result in brain injury and subsequently cerebral palsy in the child.

\section{Conclusion and policy implications}

In conclusion we found that exposure to pre-eclampsia was associated with an increased risk of cerebral palsy. However, in addition to being mediated through preterm birth, the risk for cerebral palsy was also increased in children born small for gestational age. Among children born at term, those who were exposed to pre-eclampsia and were born non-small for gestational age did not have an excess risk of cerebral palsy, and pre-eclampsia was not associated with a specific cerebral palsy subtype. These two last findings suggest that pre-eclampsia does not have a direct effect on the risk of cerebral palsy. Among very preterm children, exposure to pre-eclampsia yielded a reduced risk of cerebral palsy, but this was confined only to children who were not born small for gestational age. Delivery of a child from a pre-eclamptic pregnancy is mostly related to the health of the mother or to severe fetal growth restriction. However, our results may suggest that early signs of fetal growth deviation should be taken into account. Further studies are warranted.
We thank our colleagues at the paediatric and habilitation centres in Norway, who provided the clinical data on children with cerebral palsy.

Contributors: KMS analysed the data and wrote the manuscript. RH (obstetrician) revised the manuscript and participated in data interpretation. A-Cl and RA contributed to the research hypothesis and revision of the manuscript. GA was principally responsible for the data from the cerebral palsy registry of Norway and revised the manuscript. $S L$ reviewed and improved the statistical methods and the manuscript. LMI was principally responsible for the data from the medical birth registry of Norway and revised the manuscript. TV was responsible for research hypothesis, study design, data interpretation, and revision of the manuscript. He is the guarantor of the study and accepts full responsibility for the work and the conduct of the study. All authors had access to all of the data and approved the final version of the submitted manuscript.

\section{Funding: This research received no specific funding.}

Competing interests: All authors have completed the ICMJE uniform disclosure form at www.icmj.org/cio_disclosure.pdf (available on request from the corresponding author) and declare: no financial support from any organisation for the submitted work; no financial relationship with any companies that might have an interest in the submitted work in the previous three years; and have no non-financial interests or relationships that may be relevant to the submitted work.

Ethical approval: Written informed consent was obtained from the parents to include their children in the Norwegian Cerebral Palsy Registry and to link data to the Medical Birth Registry of Norway. The study was approved by the regional committee for medical research ethics in mid-Norway (reference No 2011/754).

Data sharing: The protocol is available on request from the corresponding author at krist@stud.ntnu.no.

1 Rosenbaum P, Paneth N, Leviton A, Goldstein M, Bax M, Damiano D, et al. A report: the definition and classification of cerebral palsy April 2006. Dev Med Child Neurol Suppl 2007;109:8-14.

2 Murphy DJ, Sellers S, MacKenzie IZ, Yudkin PL, Johnson AM. Case-control study of antenatal and intrapartum risk factors for cerebral palsy in very preterm singleton babies. Lancet 1995;346:1449-54.

3 Jarvis S, Glinianaia SV, Torrioli MG, Platt MJ, Miceli M, Jouk PS, et al. Cerebral palsy and intrauterine growth in single births: European collaborative study. Lancet 2003;362:1106-11.

4 Sibai B, Dekker G, Kupferminc M. Pre-eclampsia. Lancet 2005;365:785-99.

5 Odegard RA, Vatten LJ, Nilsen ST, Salvesen KA, Austgulen R. Preeclampsia and fetal growth. Obstet Gynecol 2000;96:950-5.

6 Gaffney G, Sellers S, Flavell V, Squier M, Johnson A. Case-control study of intrapartum care, cerebral palsy, and perinatal death. BMJ 1994;308:743-50.

7 Mann JR, McDermott S, Griffith MI, Hardin J, Gregg A. Uncovering the complex relationship between pre-eclampsia, preterm birth and cerebral palsy. Paediatr Perinat Epidemiol 2011;25:100-10

8 Thorngren-Jerneck K, Herbst A. Perinatal factors associated with cerebral palsy in children born in Sweden. Obstet Gynecol 2006;108:1499-505.

9 Greenwood C, Yudkin P, Sellers S, Impey L, Doyle P. Why is there a modifying effect of gestational age on risk factors for cerebral palsy? Arch Dis Child Fetal Neonatal Ed 2005;90:F141-6.

10 Kulak W, Okurowska-Zawada B, Sienkiewicz D, Paszko-Patej G, Krajewska-Kulak E. Risk factors for cerebral palsy in term birth infants. Adv Med Sci 2010;55:216-21.

11 Nelson KB, Ellenberg JH. Obstetric complications as risk factors for cerebral palsy or seizure disorders. JAMA 1984;251:1843-8.

12 Wu CS, Nohr EA, Bech BH, Vestergaard M, Catov JM, Olsen J. Health of children born to mothers who had preeclampsia: a population-based cohort study. Am J Obstet Gynecol 2009;201:269 e1-10.

13 Palmer L, Blair E, Petterson B, Burton P. Antenatal antecedents of moderate and severe cerebral palsy. Paediatr Perinat Epidemiol 1995;9:171-84.

14 Lee J, Croen LA, Backstrand KH, Yoshida CK, Henning LH, Lindan C, et al. Maternal and infant characteristics associated with perinatal arterial stroke in the infant. JAMA 2005;293:723-9.

15 Nelson KB. Perinatal ischemic stroke. Stroke 2007;38:742-5.

16 Surveillance of cerebral palsy in Europe: a collaboration of cerebral palsy surveys and registers. Surveillance of Cerebral Palsy in Europe (SCPE). Dev Med Child Neurol 2000;42:816-24.

17 Andersen GL, Irgens LM, Haagaas I, Skranes JS, Meberg AE, Vik T. Cerebral palsy in Norway: prevalence, subtypes and severity. Eur J Paediatr Neurol 2008;12:4-13.

18 Irgens LM. The Medical Birth Registry of Norway. Epidemiological research and surveillance throughout 30 years. Acta Obstet Gynecol Scand 2000;79:435-9.

19 Øian P, Henriksen T, Sviggum O. Hypertensive svangerskapskomplikasjoner. (Hypertensive complications of pregnancy.) (In Norwegian. No abstract available.) Veileder i Fødselshjelp:DnLF: Norwegian Med Assoc 1998:94-7.

20 Skjaerven R, Gjessing HK, Bakketeig LS. Birthweight by gestational age in Norway. Acta Obstet Gynecol Scand 2000;79:440-9.

21 Victora CG, Huttly SR, Fuchs SC, Olinto MT. The role of conceptual frameworks in epidemiological analysis: a hierarchical approach. Int J Epidemiol 1997;26:224-7. 


\section{What is already known on this topic}

Pre-eclampsia is a frequent cause of preterm birth and being born small for gestational age, both of which are known risk factors for cerebral palsy

Observational studies have shown conflicting results with respect to whether pre-eclampsia is a risk factor for cerebral palsy

\section{What this study adds}

Pre-eclampsia is a risk factor for cerebral palsy mainly mediated through preterm birth and being small for gestational age Among term born children exposed to pre-eclampsia only those born small for gestational age had an excess risk of cerebral palsy Pre-eclampsia was not associated with specific subtypes of cerebral palsy

22 Weinberg CR. Toward a clearer definition of confounding. Am J Epidemiol 1993;137:1-8.

23 VanderWeele TJ, Hernandez-Diaz S. Is there a direct effect of pre-eclampsia on cerebral palsy not through preterm birth? Paediatr Perinat Epidemiol 2011;25:111-5.

24 Thomsen LCV, Klungsøyr K, Roten LT, Tappert C, Araya E, Bærheim G, et al. Validity of the diagnosis of pre-eclampsia in the Medical Birth Registry of Norway. Acta Obstet Gynecol Scand 2013; published online 26 April, doi:10.111/aogs.12159.

25 ACOG practice bulletin. Diagnosis and management of preeclampsia and eclampsia. Number 33, January 2002. Obstet Gynecol 2002:99:159-67.

26 Gardosi J, Francis A. A customized standard to assess fetal growth in a US population. Am J Obstet Gynecol 2009;201:25 e1-7.

27 Stoknes M, Andersen GL, Elkamil Al, Irgens LM, Skranes J, Salvesen KA, et al. The effects of multiple pre- and perinatal risk factors on the occurrence of cerebral palsy. A Norwegian register based study. Eur J Paediatr Neurol 2012;16:56-63.

28 Spinillo A, Capuzzo E, Cavallini A, Stronati M, De Santolo A, Fazzi E. Preeclampsia, preterm delivery and infant cerebral palsy. Eur J Obstet Gynecol Reprod Biol 1998;77:151-5.

29 Wilson-Costello D. Risk factors for neurologic impairment among very low-birth-weight infants. Semin Pediatr Neurol 2001;8:120-6.
30 Dammann O, Kuban KC, Leviton A. Perinatal infection, fetal inflammatory response, white matter damage, and cognitive limitations in children born preterm. Ment Retard Dev Disabil Res Rev 2002:8:46-50.

31 McElrath TF, Allred EN, Boggess KA, Kuban K, O'Shea TM, Paneth N, et al. Maternal antenatal complications and the risk of neonatal cerebral white matter damage and late cerebral palsy in children born at an extremely low gestational age. Am J Epidemiol 2009;170:819-28.

Accepted: 18 June 2013

\section{Cite this as: BMJ 213;347:f4089}

This is an Open Access article distributed in accordance with the Creative Commons Attribution Non Commercial (CC BY-NC 3.0) license, which permits others to distribute, remix, adapt, build upon this work non-commercially, and license their derivative works on different terms, provided the original work is properly cited and the use is non-commercial. See: http://creativecommons.org/licenses/by-nc/3.0/. 


\section{Tables}

Table 1| Maternal and pregnancy related characteristics reported as number (percentage) in children with or without exposure to pre-eclampsia and with or without cerebral palsy born in Norway 1996-2006

$$
\text { No pre-eclampsia Pre-eclampsia }
$$

Characteristics

No cerebral palsy $(n=593777)$ Cerebral palsy $(n=774)$ No cerebral palsy $(n=22881)$ Cerebral palsy $(n=75)$

\begin{tabular}{|c|c|c|c|c|}
\hline Para 0 & $235858(39.7)$ & $343(44.3)$ & $13768(60.2)$ & $52(69.3)$ \\
\hline Smoker in pregnancy & $76095(22.6)$ & $106(23.9)$ & $2642(19.2)$ & $12(25.0)$ \\
\hline Assisted fertilisation & $7024(1.2)$ & $12(1.6)$ & $380(1.7)$ & $5(6.7)$ \\
\hline \multicolumn{5}{|l|}{ Child characteristics } \\
\hline Male & $304574(51.3)$ & $448(57.9)$ & $11865(51.9)$ & $50(66.7)$ \\
\hline Small for gestational age & $43399(7.5)$ & $111(15.1)$ & $4523(20.3)$ & $31(44.3)$ \\
\hline Term birth ( $\geq 37$ weeks) & $549172(95.4)$ & $504(67.5)$ & $17002(76.3)$ & $21(29.2)$ \\
\hline SGA infants born at term & $40854(7.4)$ & $76(15.4)$ & $2676(15.8)$ & 7 (33.3) \\
\hline Moderate preterm birth (32-36 weeks) & $23185(4.0)$ & $106(14.2)$ & $4092(18.4)$ & $21(29.2)$ \\
\hline SGA infants born moderately preterm & $2206(9.5)$ & $19(17.9)$ & $1363(33.3)$ & $9(42.9)$ \\
\hline Very preterm birth (<32 weeks) & $3216(0.6)$ & $137(18.3)$ & $1180(5.3)$ & $30(41.7)$ \\
\hline SGA infants born very preterm & $339(10.6)$ & $16(11.9)$ & $484(41.2)$ & $15(53.6)$ \\
\hline
\end{tabular}

Maternal characteristics

$\mathrm{SGA}=$ small for gestational age.

18841 children had missing data on gestational age, 19460 children had missing data on small for gestational age status. 
Table 2/ Unadjusted (model 1) and adjusted odds ratios for cerebral palsy after exposure to pre-eclampsia

\begin{tabular}{|c|c|c|c|}
\hline \multirow[b]{2}{*}{ Potential mediators } & \multicolumn{3}{|c|}{ Odds ratios $(95 \% \mathrm{Cl})$} \\
\hline & Model $1^{*}$ & Model 2† & Model 3‡ \\
\hline Pre-eclampsia & 2.52 (1.98 to 3.19 ) & 2.14 (1.67 to 2.74 ) & $0.73(0.56$ to 0.96$)$ \\
\hline Small for gestational age & - & 2.30 (1.91 to 2.76 ) & 1.90 (1.58 to 2.30$)$ \\
\hline \multicolumn{4}{|l|}{ Duration of pregnancy: } \\
\hline $37-40$ weeks & - & - & 1.00 (reference) \\
\hline $32-36$ weeks & - & - & $5.10(4.18$ to 6.20$)$ \\
\hline$<32$ weeks & - & - & 40.71 (33.70 to 49.17$)$ \\
\hline
\end{tabular}

*Unadjusted odds ratio for association between pre-eclampsia and cerebral palsy. †Adjusted for small for gestational age.

$\ddagger$ Adjusted for small for gestational age and duration of pregnancy. 
Table $3 \mid$ Prevalence and odds of cerebral palsy according to exposure to pre-eclampsia and small for gestational age, stratified by duration of pregnancy

Duration of pregnancy and exposure Total No at risk Cerebral palsy No cerebral palsy No of cases of cerebral palsy/1000 Odds ratio $(95 \% \mathrm{Cl})$ $\geq 37$ weeks

\begin{tabular}{|c|c|c|c|c|c|}
\hline \multicolumn{6}{|c|}{ Non-small for gestational age: } \\
\hline No pre-eclampsia & 508228 & 418 & 507810 & 0.8 & 1.0 (reference) \\
\hline Pre-eclampsia & 14323 & 14 & 14309 & 1.0 & 1.19 (0.70 to 2.03$)$ \\
\hline \multicolumn{6}{|c|}{ Small for gestational age: } \\
\hline No pre-eclampsia & 40930 & 76 & 40854 & 1.9 & 2.26 (1.77 to 2.89$)$ \\
\hline Pre-eclampsia & 2683 & 7 & 2676 & 2.6 & $3.18(1.50$ to 6.71$)$ \\
\hline \multicolumn{6}{|l|}{ 32-36 weeks } \\
\hline \multicolumn{6}{|c|}{ Non-small for gestational age: } \\
\hline No pre-eclampsia & 21027 & 87 & 20940 & 4.1 & 1.0 (reference) \\
\hline Pre-eclampsia & 2736 & 12 & 2724 & 4.4 & 1.06 (0.58 to 1.94$)$ \\
\hline \multicolumn{6}{|c|}{ Small for gestational age: } \\
\hline No pre-eclampsia & 2225 & 19 & 2206 & 8.5 & $2.07(1.26$ to 3.41$)$ \\
\hline Pre-eclampsia & 1372 & 9 & 1363 & 6.6 & $1.59(0.80$ to 3.16$)$ \\
\hline \multicolumn{6}{|l|}{$<32$ weeks } \\
\hline \multicolumn{6}{|c|}{ Non-small for gestational age: } \\
\hline No pre-eclampsia & 2964 & 119 & 2845 & 40.1 & 1.0 (reference) \\
\hline Pre-eclampsia & 705 & 13 & 692 & 18.4 & $0.45(0.25$ to 0.80$)$ \\
\hline \multicolumn{6}{|c|}{ Small for gestational age: } \\
\hline No pre-eclampsia & 355 & 16 & 339 & 45.1 & 1.13 (0.66 to 1.93$)$ \\
\hline Pre-eclampsia & 499 & 15 & 484 & 30.1 & 0.74 (0.43 to 1.28$)$ \\
\hline \multicolumn{6}{|c|}{ Missing data on gestational age: } \\
\hline No pre-eclampsia & 18231 & 27 & 18204 & 1.5 & 1.0 (reference) \\
\hline Pre-eclampsia & 610 & 3 & 607 & 4.9 & 3.33 (1.01 to 11.01$)$ \\
\hline
\end{tabular}


Table 4 | Odds for cerebral palsy according to exposure to pre-eclampsia and duration of pregnancy, compared with reference group of children unexposed to pre-eclampsia and born at term (model 4, adjusted for small for gestational age)

\begin{tabular}{llc} 
Pre-eclampsia & Duration of pregnancy & Adjusted odds ratio (95\% Cl) \\
Absent & Term & 1.00 (reference) \\
\hline Present & Term & $1.28(0.83$ to 1.98$)$ \\
\hline Absent & Moderately preterm & $4.99(4.05$ to 6.16$)$ \\
\hline Present & Moderately preterm & $4.64(2.98$ to 7.23$)$ \\
\hline Absent & Very preterm & $45.90(37.81$ to 55.72$)$ \\
\hline Present & Very preterm & $20.37(13.74$ to 30.22$)$ \\
\hline Small for gestational age & - & $1.95(1.61$ to 2.36$)$ \\
\hline
\end{tabular}

